\title{
Nachruf auf eine Ausbildungsreform
}

Der Rechtsausschuß des Deutschen Bundestages hat auf seinen Sitzungen vom 6. und 13. Mai seine Beschlüsse zur Reform der Juristenausbildung (Änderung des $\$ s$ DtRiG) gefaßt. Diese Beschlüsse, die noch im Juni in 2. und 3. Lesung vom Bundestag verabschiedet werden sollen, bringen ebenso überraschende wie schwerwiegende Veränderungen gegenüber der bisherigen Konzeption der Ausbildungsreform. Sollten diese Beschlüsse, wie vorgesehen, in Kürze Gesetzeskraft erhalten, so bleibt nur noch das Ende der Ausbildungsreform « zu verkünden: Die Leichenfeier findet in privaten Rechtsschulen statt. Doch Zynismus wäre nur die bürgerliche Form der Reaktion auf das jüngste Lehrstück des bundesrepublikanischen $*$ Reform «kapitalismus und seines Parlaments, in dessen letztem Akt die weitreichenden Erwartungen engagierter Reformer sich als pure Illusionen entpuppen und ihre umfangreichen Aktivitäten ihres Surrogatcharakters für politische Praxis überführt werden.

\section{Entscheidungen des Rechtsausschusses}

Schon der in 1. Lesung an den Rechtsausschuß verwiesene Entwurf der Bundesregierung (Jahn-Entwurf) hatte deutlich gemacht, daß an eine allgemeine Juristenausbildungsreform nicht gedacht war: die zweiphasige Ausbildung wurde abgesehen von einer inhaltlich belanglosen Kürzung des Referendardienstes auf 2I Monate - in unveränderter Form als Regelausbildung beibehalten; die Durchführung der Einphasenausbildung (als Organisationsmodell zur Integration von Theorie und Praxis, Rechts- und Sozialwissenschaften) sollte unter Verzicht auf eine folgenreiche politische Entscheidung als zeitlich befristetes Experiment der eigenverantwortlichen Regie der Länder überlassen bleiben, die Qualität der konkurrierenden Reformmodelle nach den Chancen der ausgebildeten Juristen auf dem Stellenmarkt bemessen werden. Doch der sonst so gepriesene Marktmechanismus schien dem Rechtsausschuß trotz des fehlenden demokratischen Charakters der Verwertungsinstanzen juristisch Qualifizierter (Justiz, Verwaltung, Wirtschaft) keine ausreichende Garantie für eine systemkonforme Juristenausbildung zu bieten: Die »Experimentierklausel « des $\$$ sa DtRiG liest sich nunmehr wie die bornierte Anweisung zur Einrichtung von »gleidhwertigen« Kurzstudien: $\gg$ Das Landesrecht kann Studium und praktische Vorbereitung zu einer gleichwertigen Ausbildung von mindestens $5^{1 / 2}$ Jahren zusammenfassen. Ein Teil der Ausbildung ist bei (!) Gerichten, Verwaltungsbehörden und Rechtsanwälten abzuleisten. Die erste Prüfung kann durch eine $Z$ wischenprüfung oder durch ausbildungsbegleitende Leistungskontrollen ersetzt werden. Die zweite Prüfung soll in ihren Anforderungen der in $\ \mathcal{S}$ vorgesehenen zweiten Prüfung gleichwertig (!) sein.... State einer qualitativen Neubestimmung der Ausbildungsinhalte, wie 
sie - in welch rudimentärer Form auch immer - sämtlichen Einphasenmodellen zugrunde lag und in der Forderung nach Integration von Rechts- und Sozialwissenschaften ihren einheitlichen Ausdruck fand, geht es nach der Vorlage des Rechrsausschusses nur mehr um eine zeit- und kostensparende "Zusammenfassung " von Studium und praktischer Vorbereitung. Der erhöhten Intensität der Ausbildung entspricht die Verschulung durch Einführung der Zwischenprüfung. Gemäß diesen Bestrebungen zur Blockierung nicht examens- und verwertungsorientierter Lerninteressen wurde auch die zweiphasige Ausbildung »reformierr«: Die Stationsnoten des auf 2 Jahre gekürzten Referendardienstes sind bundeseinheitlich zu 33\% auf die Note im 2. Stantsexamen anzurechnen. Das bedeuter: Examensdruck in Permanenz bei identischen Ausbildungsinhalten.

Doch die gravierendste Änderung gegenüber der bisherigen Ausbildung und sämtlichen offiziellen »Reform《entwürfen verbirgt sich hinter der scheinbar harmlosen Formulierung des neu hinzugenommenen $₫ s b$ Abs. 2: „Eine erfolgreich abgeschlossene Teilnahme an einer von der Landesregierung anerkannten Ausbildung, die auf die Vorbereitung für die erste Prüfung ausgerichtet ist, kann auf Antrag bis zur Dauer von I2 Monaten auf die Ausbildung ... angerechner werden. « Mit der Annahme dieser Bestimmung hat der Rechrsausschuß Bestrebungen legalisiert, auf die die Offentlidhkeir erstmals durch einen Artikel der FAZ v. 17. 2. 1971 hingewiesen worden war, in der der CDU-Bundestagsabgeordnete Hans Dichgans, Berichterstatter des Rechtsausschusses für Fragen der Reform der juristischen Ausbildung, Mitglied des $*$ Bundes Freiheit der Wissenschaft « und Verbandsgeschäfrsführer der $\$$ Wirtschaftsvereinigung Stahl und Eisen«, seine Auffassungen zur Neugestaltung der Juristenausbildung dargelegt hatte: die Errichtung privater Rechtsschulen.

Bereits damals wußte die FAZ zu konstatieren: "So schodkierend der Gedanke der privaten Rechtsschulen auf den ersten Blick scheint, so wenig ist Dichgans hier Einzelkämpfer. Er hat manche Zustimmung bei den Juristen seiner Fraktion gefunden - und überraschenderweise auch bei Juristen der SPD.« Er hat. Die Beschlüsse des Rechtsausschusses wurden einstimmig gefaßt.

$\mathrm{Da} B$ die vom Rechtsausschuß durchgeführte umfangreiche Fragebogenaktion zur Reform der Juristenausbildung, mit der zahlreiche Stellungnahmen von Gerichten, Ministerien, Fakultäten, Berufs-, Wirtschafts- und Kommunalverbänden eingeholt worden waren, ebenso wie das am 25. 3. in Bonn veranstaltete öffentliche Hearing eher zur Irreführung der Offentlichkeit und der Illusionierung der Betroffenen als zur Meinungsbildung der Abgeordneten dienten, mag für liberale Parlamentarismuskritiker noch eigens betont werden: zur Unterstützung der Idee der privaten Rechtsschulen fand sich auf dem Hearing nur Herr. Rechtsanwalt Dr. jur. Paul Schneider, Repetitor in Bonn, bereit; in den 78 ausgewerteten Fragebögen findet sich nur ein Votum (nschwaches Ja") für den Vorschlag der Rechtsschule, die offizielle Eingliederung des Repetitors in das juristische Studium wurde »in relativ wenigen Fällen... überhaupt befürwortet «. Der zweite Punkt, in dem der Rechrsausschuß eine eigene politische Entscheidung unabhängig von den vorliegenden Entwürfen fällte, die 1/3 Anrechnung von Stationsnoten auf das 2. Staatsexamen, stand erst gar nicht zur Diskussion.

\section{Provisorische Interpretation}

Diese Beschlüsse paralysieren die größte Hoffnung der Reformer: die Einphasenausbildung. Das Programm der Einphasenausbildung, mit den sie konstituierenden Prinzipien der Zusammenfassung von theoretischer und praktischer Ausbil- 
dung und der Integration der Rechtswissenschaft in die Sozialwissenschaften, war auf dem Feld der Juristenausbildung die konziseste und reflektierteste Antwort auf die obsolet gewordenen Ausbildungsformen und -inhalte. In dem durch seine Bremer Fassung reformulierten sog. Loccumer Modell stand sie auf dem Boden einer durch Habermas, Offe, Etzioni u. a. belehrten funktionalen Systemtheorie. Das Element an "kritischer Reflexion", das hiermit eingeführt war, enthielt minimale Transformations- und Kritikchancen, die zugleich Gefahren für eine Sozialtechnologie darstellen mußten, die sich letzrlich in der Anpassung sämtlicher Gesellschaftsteilsysteme an das naturwüchsige wireschaftliche Subsystem erschöptt. Die Beschlüsse des Rechtsausschusses strangulieren derartige Einphasenmodellierungen in nuce durch den Einbau institutioneller Sprengkapseln in Form von unsinnigen Examensvorschriften ( $\$$ s a I). Das Dilemma des Frankfurter Reformprojekts - in einem ernsthafte Reformen erstidkenden institutionellen Rahmen arbeiten zu müssen - wird für die Einphasenmodelle bundesweit reproduziert. Unter diesen Vorzeichen müssen die Versuche in Einphasenausbildung dazu verkümmern, Reform ausschließlich in terms von Effizienzsteigerung und Kostenersparnis zu betreiben.

Die Beschlüsse des Rechtsausschusses ersetzen Reform traditioneller Juristenausbildung durch Verkürzung des Referendariats um ein halbes Jahr. Hier sucht sich offensichtich Haushaltspolitik als Juristenausbildungsreform zu kamouflieren. Bei Aufrechterhaltung des 2. Staatsexamens in seiner bisherigen Form und 1/s Anrechnung der Stationsnoten bedeutet dies eine Perpetuierung des bisherigen Zustands auf dequalifizierterem Niveau, nämlich weil noch mehr verschult und auf irrationale Examensanforderungen konzentriert.

Die Beschlüsse des Rechtsausschusses bescheinigen zudem den alten juristischen Fakultäten, daß sie ihre Aufgabe mehr schlecht als recht erfüllen, indem sie die bisherige Ausbildung für ergänzungsbedürftig erachten durch extrauniversiräre Ausbildung an privaten Rechtsschulen. Die Zulassung extrauniversitärer Juristenausbildung ist denn auch die eigentliche Reformpointe dieser Beschlüsse. Die privaten Rechtsschulen lassen sich dann bequem integrieren in die privaten Stiftungsuniversitäten, wie sie unbenannt im Regierungsentwurf eines Hochschulrahmen $G$ vorgesehen sind. ${ }^{t}$

Diese Beschlüsse des Rechtsausschusses werden erst recht verständlich vor dem Hintergrund des allgemeinen - für die BRD darakteristischen - strategischen Konzepts von Gesellschaftsreform bzw. gesellschaftl. Evolution. Dieses Konzept ist an »entideologisierter Gesellschaftstheorie " orientiert, als deren Prototyp in den westlichen spärkapitalistischen Industriegesellschaften heure die Systemrheorie auftritt, und es teilt deren methodologische und inlaltliche Beschränkungen. Sozialer Wandel und Fortschritr sind nur noch begreifbar in den Kategorien der strukturellen bzw. funkrionalen Differenzierung. Die Beschlüsse des Bundestagsrechtsausschusses unternehmen es nun - und dies haben sie wiederum mit dem Leussink-Entwurf eines HochschulrahmenG gemeinsam -, die »überfällige* Ausdifferenzierung akademische Lehr- und Lernfunkrionen in Angriff zu nehmen. Ist die Ausgliederung der Forschung aus den Universitäten bereits ein auch in das Bewußtsein der Offentlichkeit gedrungenes Faktum, beginnt jetzt die Evakuierung der Lehre aus dem Bereich, in dem sie bis dato monopolisiert war, dem der autonomen staatlichen Universitäten. Die Privatisierung der akademi-

1 Vergl. zu den privaten Stiftungsuniversitäten m. ausf. Nachweisen L. Niemann KJ r970, 2 I f. An dieser Stelle hervorzuheben ise das - Plädoyer für eine Industriehochschulea des Schöpfers der privaten Rechtsschule Hans Dichgans, publik 7. 27. 3. 1970. 
schen Lehrfunktionen sucht sich als rational, demokratisch und pluralistisch zu legitimieren. Sie ist alles dies nicht. Sie ist primär Reaktion auf die sog. Demokratisierung und Politisierung der Universitäten, die u. a. dazu geführt hat, daß die Universitäten unkontrollierbaren gesellschaftlichen und ökonomischen Interessen nicht mehr ohne weiteres verfügbar sind. Privatisierung staatlicher Funktionen meine denn auch dies, durch Ausschalten von Offentlichkeit und demokratischer Kontrolle das Reflexivwerden und damit à la longue das Dysfunktionalwerden wichtiger gesellschaftlicher Prozesse, die nicht ohne weiteres substituierbar sind, zu verhindern. Privatisierung staatlicher Funktionen verkauft sich als (system-) rational, in Wahrheit institutionalisiert sie die Irrationalität, weil sie die Reflexion auf die strukturelle Pathologie der spätkapitalistischen Gesellschaft, deren Symptom sie ist, abschneidet.

Thomas Blanke Gert Brüggemeier

\section{Aus dem Inhalt der nächsten Hefte:}

W. Abendroth, Staatsgewalt und Rechtsordnung im Rahmen der Transformation in eine sozialistische Gesellschaft - O.Negt, Genesis und Geltung. Zur Rechtstheorie bei Kant und Mars - K. Lenk, Programm einer Rechtssoziologie in kritischer Absicht - F. Sack, Selektion und Kriminalität - Ch. Müller, H. Heilers Beitrag zu einer Theorie der politischen Entscheidung - U. Mäckenberger, Privatrechtsideologie - D. Hart, Zur Funktion Allgemeiner Geschäftsbedingungen $-L$. Unterseher, Tarifliche Schlichtung: ein ökonomisches Gerichtsverfahren $-H$. Rottleutbner, Was heißt Klassenjustiz? 